University of Nebraska - Lincoln

DigitalCommons@University of Nebraska - Lincoln

Timothy J. Gay Publications

Research Papers in Physics and Astronomy

2-1-2007

\title{
Radiation trapping in rubidium optical pumping at low buffer-gas pressures
}

Mark A. Rosenberry

Siena College, mrosenberry@siena.edu

J. P. Reyes

Siena College, Loudonville, New York

D. Tupa

Los Alamos National Laboratory, Los Alamos, New Mexico

Timothy J. Gay

University of Nebraska - Lincoln, tgay1@unl.edu

Follow this and additional works at: https://digitalcommons.unl.edu/physicsgay

Part of the Physics Commons

Rosenberry, Mark A.; Reyes, J. P.; Tupa, D.; and Gay, Timothy J., "Radiation trapping in rubidium optical pumping at low buffer-gas pressures" (2007). Timothy J. Gay Publications. 49.

https://digitalcommons.unl.edu/physicsgay/49

This Article is brought to you for free and open access by the Research Papers in Physics and Astronomy at DigitalCommons@University of Nebraska - Lincoln. It has been accepted for inclusion in Timothy J. Gay Publications by an authorized administrator of DigitalCommons@University of Nebraska - Lincoln. 


\title{
Radiation trapping in rubidium optical pumping at low buffer-gas pressures
}

\author{
M. A. Rosenberry, ${ }^{1}$ J. P. Reyes, ${ }^{2}$ D. Tupa, ${ }^{3}$ and T. J. Gay ${ }^{2}$ \\ ${ }^{1}$ Siena College, Loudonville, New York 12211, USA \\ ${ }^{2}$ Behlen Laboratory of Physics, University of Nebraska, Lincoln, Nebraska 68588-0111, USA \\ ${ }^{3}$ Los Alamos National Laboratory, Los Alamos, New Mexico 87545, USA
}

(Received 22 September 2006; published 1 February 2007)

\begin{abstract}
We have made a systematic study of rubidium optical pumping in a simple cylindrical cell geometry with a high-power $10 \mathrm{~W}$ diode laser array, low magnetic fields, and buffer-gas pressures of less than 50 torr. We have determined rubidium polarizations experimentally for $\mathrm{H}_{2}, \mathrm{~N}_{2}, \mathrm{He}$, and Ar buffer gases, with $\mathrm{Rb}$ number densities from $10^{12}$ to $10^{13} \mathrm{~cm}^{-3}$. Comparison to a relatively simple optical pumping model allows us to extract useful information about radiation trapping and quenching effects.
\end{abstract}

DOI: 10.1103/PhysRevA.75.023401

PACS number(s): $32.80 . \mathrm{Bx}$

\section{INTRODUCTION}

Optical pumping of alkali-metal atoms is an important tool in atomic physics. It is used in a broad range of experiments such as the storage of light [1], atomic clocks [2,3], and the generation of polarized noble gases through spin exchange [4]. Although the fundamental technique of these experiments is the same, they operate under very different conditions. Storage of light in a warm rubidium vapor is done with little or no buffer gas and spectrally narrow, lowpower lasers, while systems that use spin-exchange optical pumping to generate polarized noble gases tend to use high buffer-gas densities and broad, high-power lasers. The work reported here deals with a third regime, using broad, highpower lasers with low pressures of buffer gas. These conditions are required by alcali-metal atoms "spin-filter" sources of polarized electrons [5], which are useful for studies of phenomena such as collisionally induced atomic multipole moments [6].

In spin-filter type sources, electrons become polarized by spin exchange with the optically pumped rubidium atoms, so high rubidium polarizations are crucial. However, the necessity of passing the electrons through the $\mathrm{Rb}$ vapor requires that the buffer gas pressures be modest (generally less than a few torr) to maintain any appreciable electron current. Furthermore, to make the system as simple as possible, we currently use a diode laser array rather than a dye laser. This results in conditions that are unusual for modern optical pumping: a spectrally broad light source pumping an optically thick sample without sufficient molecular buffer gas to completely quench the excited-state rubidium.

\section{EXPERIMENTAL PROCEDURE AND MEASUREMENT}

To better understand our system performance, we undertook a study to characterize the rubidium polarization under these conditions. Our optical pumping experiments were conducted in a test cell with no electron beam present (Fig. 1).

The cell is a modified 2-3/4" Conflat nipple, 5" long, with standard glass view ports at each end and a side connection to the vacuum system. The vacuum is maintained using a diffusion pump, and the buffer-gas pressures are de- termined by a capacitance manometer. A Rb ampoule (natural abundance) is placed directly in the test cell. For our uncoated cell, exchange with the bulk material is a negligible source of polarization loss. The system is placed inside a solenoid that provides a magnetic field along the pumping axis.

A set of heater tapes is used to maintain the temperature of the cell to roughly $1{ }^{\circ} \mathrm{C}$ accuracy, at a specific buffergas pressure. To make measurements at a constant rubidium density, this temperature had to be adjusted as the buffer-gas pressure was varied, since the change in pressure leads to greater absorption of laser light and varying thermal gradients in the cell. A heat gun directed at the front window of the cell keeps it at a higher temperature than the metal sides to prevent condensation of the rubidium on the glass.

The rubidium is pumped using a $10 \mathrm{~W}$ diode array tuned to the rubidium $D 1$ transition, and has a spectral width of about $700 \mathrm{GHz}$. Given our operating conditions, about $2 \mathrm{~W}$ of laser power actually reaches the cell, with a rectangular shape (roughly $4.0 \times 0.5 \mathrm{~cm}$ ) as determined by our optical setup. From these values, we deduce that we are well below saturation for this transition. The average density and polar-

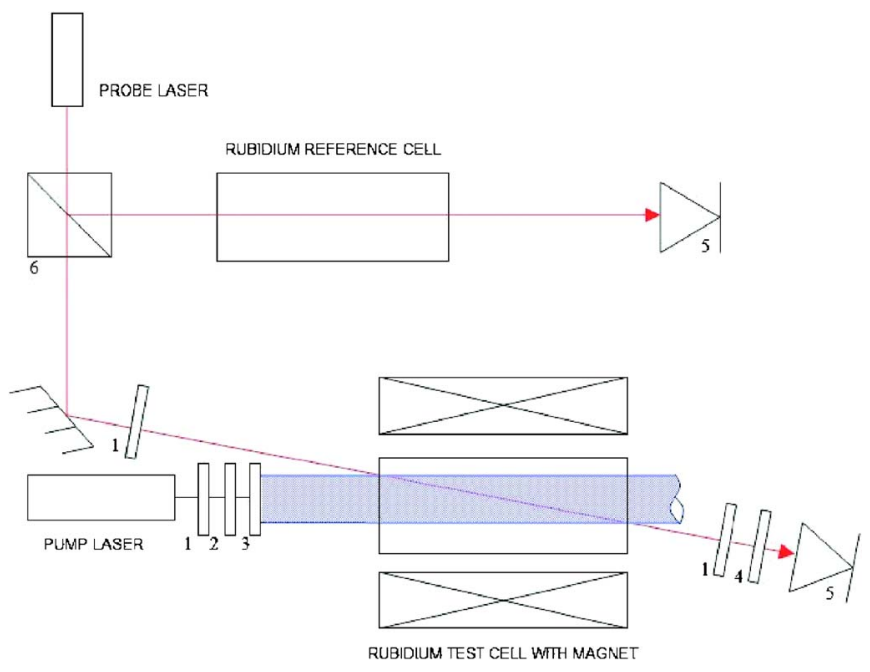

FIG. 1. (Color online) Apparatus schematic. (1) linear polarizers, (2) quarter-wave retarder, (3) telescope, (4) interference filter, (5) photodiode, (6) beamsplitter. 
ization of the $\mathrm{Rb}$ are determined optically by measuring the Faraday rotation of a linearly polarized probe laser at the $\mathrm{Rb}$ $D 2$ transition. The probe beam is oriented so that it overlaps the pump laser throughout the cell's interior. The photodetector that observes the probe signal is shielded by a spectrally narrow interference filter to block out the pump light. To measure the detuning of the probe beam from resonance, $\delta$, a room-temperature reference cell is used for comparison; typically measurements were made at $\delta=6.0 \mathrm{GHz}$ detuning.

To measure the number density of the rubidium, $n_{\mathrm{Rb}}$, the magnetic field is temporarily increased to $180 \mathrm{G}$. Optical pumping and polarization measurements are performed at the lower field of about $10 \mathrm{G}$. When the magnetic field is $180 \mathrm{G}$, the optical rotation of linearly polarized light is dominated by the diamagnetic Faraday effect. Measuring the optical rotation $\Delta \phi$ associated with this effect yields the density $[7,8]$,

$$
n_{\mathrm{Rb}}=\frac{48 \pi(\Delta \phi) \delta^{2}}{7 L \Gamma_{\mathrm{nat}} \lambda_{2}{ }^{2}\left(\mu_{B} / h\right) B},
$$

where $L$ is the cell length, $\lambda_{2}$ is the $D 2$ wavelength of $780.0 \mathrm{~nm}, \Gamma_{\text {nat }}$ is the $D 2$ natural linewidth of $6.1 \mathrm{MHz}$, and $\left(\mu_{\mathrm{B}} / h\right)$ is the Bohr magneton of $1.4 \mathrm{MHz} / \mathrm{G}$. For a $10 \mathrm{G}$ magnetic field, the optical rotation is dominated by the paramagnetic Faraday effect, and yields the $\mathrm{Rb}$ polarization $[7,8]$

$$
P_{\mathrm{Rb}}=\frac{56 \pi(\Delta \phi) \delta}{3 n_{\mathrm{Rb}} L \Gamma_{\mathrm{nat}} \lambda_{2}{ }^{2}},
$$

where $P_{\mathrm{Rb}}$ is defined as

$$
P_{\mathrm{Rb}}=\frac{n_{\uparrow}-n_{\downarrow}}{n_{\uparrow}+n_{\downarrow}},
$$

with $n$ referring to the population densities of the rubidium valence electron spins along the pumping axis. We emphasize that the polarizations shown are the average value within the pump beam volume over the cell length.

Our data covers four different rubidium densities, from $1.0 \times 10^{12} \mathrm{~cm}^{-3}$ to $1.0 \times 10^{13} \mathrm{~cm}^{-3}$, and four different buffergas species: argon, helium, hydrogen, and nitrogen. There is some variation in rubidium density during each run or between buffer species, at the 10-20\% level. The measured values of the rubidium polarization can be seen in Figs. 2(a)-2(h), as well as curves based on our simplified model. Data obtained with no buffer-gas pressure is presented separately in Fig. 3.

\section{DESCRIPTION OF MODEL}

We present a relatively simple model for our apparatus to describe the rubidium polarization in the presence of buffer gas. The polarization will depend on a number of factors, including the laser power absorbed, the diffusion rate of rubidium through the buffer gas to the walls (assumed to be completely depolarizing), polarization losses due to collisions with the buffer-gas atoms, the effects of radiation trapping, and the mitigation of trapping from quenching collisions with molecular buffer gases. We begin with a standard equation for the rubidium polarization,

$$
P_{\mathrm{Rb}}=\frac{\gamma}{\gamma+\Gamma},
$$

where $\gamma$ describes the integrated optical pumping rate, and $\Gamma$ represents the sum of all spin destruction mechanisms. We write the loss rate $\Gamma$ as the sum of three terms,

$$
\Gamma=\Gamma_{\text {dif }}+\Gamma_{\text {col }}+\Gamma_{\mathrm{rt}},
$$

where $\Gamma_{\text {dif }}$ is the contribution from rubidium diffusing to the walls, $\Gamma_{\text {col }}$ is from collisions in the gas, and $\Gamma_{\mathrm{rt}}$ is from radiation trapping. The first two loss mechanisms are well understood; for our situation, we calculate that relaxation due to the diffusion to the walls [7] is much greater than relaxation from buffer-gas collisions $[9,10]$, and so we neglect contributions of collisions to spin relaxation.

For a cylindrical cell of radius $R$, diffusive losses are approximated by the lowest order diffusion mode, leading to a loss rate of [7]

$$
\Gamma_{\mathrm{dif}}=\left[\left(\frac{\pi}{L}\right)^{2}+\left(\frac{\mu}{R}\right)^{2}\right] D_{\mathrm{o}} \frac{p_{o}}{p},
$$

where $\mu=2.405$ is the first zero of the Bessel function $J_{o}, D_{o}$ is the diffusion constant at $p_{o}=760$ torr, and $p$ is the cell pressure in torr. The values of the diffusion constants at $100{ }^{\circ} \mathrm{C}$ are $0.22 \mathrm{~cm}^{2} / \mathrm{s}$ for $\operatorname{Ar}$ [9], $0.23 \mathrm{~cm}^{2} / \mathrm{s}$ for $\mathrm{N}_{2}$ [11], $0.73 \mathrm{~cm}^{2} / \mathrm{s}$ for $\mathrm{He}$ [12], and $1.52 \mathrm{~cm}^{2} / \mathrm{s}$ for $\mathrm{H}_{2}$ [13].

Radiation trapping at low magnetic fields is quite complex and difficult to calculate [14-17]. Radiation trapping occurs in an optically thick vapor when photons spontaneously emitted during the optical pumping cycle are absorbed by other atoms before the photons escape the sample. This process is harmful to polarization in two ways. For any value of the magnetic field, it can increase the average time atoms spend in their excited state per pumping cycle, thus effectively reducing the pump rate. In addition, in low magnetic fields, reabsorbed photons may depolarize the absorbing atoms, so the relaxation rate is increased.

It can be shown that a multiplicity factor, $M$, can effectively characterize the extent of radiation trapping. Physically, $M$ is the average number of times a photon is emitted before it escapes the experiment volume; $(M-1)$ is the average number of times a photon is absorbed by atoms after it is produced in a pumping cycle. For optically thin samples, trapping does not occur and the value of $M$ approaches 1 .

It can also be shown that $M$ is useful in describing the effects of radiation trapping on optical pumping even if calculations of $M$ are greatly simplified. For example, it is possible to neglect complicated contributions such as the angular emission of radiation, and to use an overall number density of atoms rather than carefully accounting for the number of atoms in excited and ground states in an iterative computation. It is also adequate to approximate the multiplicity factor for our cylindrical geometry with that of a sphere of the same radius. A detailed derivation of the multiplicity factor and examination of these simplifying assumptions is presented elsewhere [18].

Briefly, the simplified multiplicity factor is derived from the rate equations for the number densities of atoms in the ground and excited states, $n_{\mathrm{g}}$ and $n_{\mathrm{e}}$ respectively, in a two- 

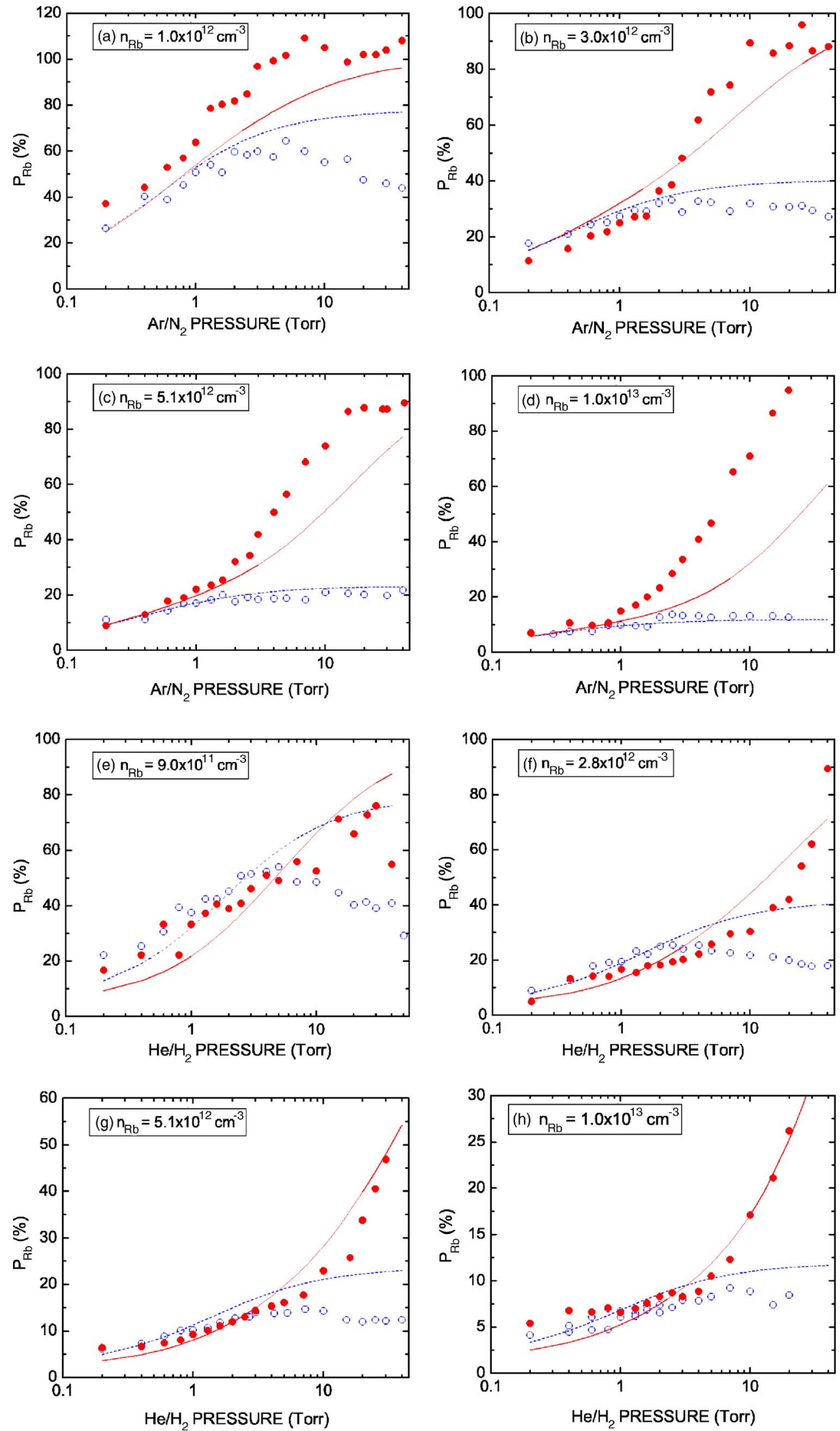

FIG. 2. (Color online) (a)-(h) Rubidium polarization for different rubidium densities as a function of buffer gas pressure. (a)-(d) show results for argon and nitrogen, and (e)-(h) show results for hydrogen and helium. The experimental data is shown with filled circles for the results with molecular buffer gas and open circles for the atomic gases. The solid line is the result of our model with molecular buffer gas and the dashed line with the atomic gas. Note the different vertical scale for (a), (g), and (h). 


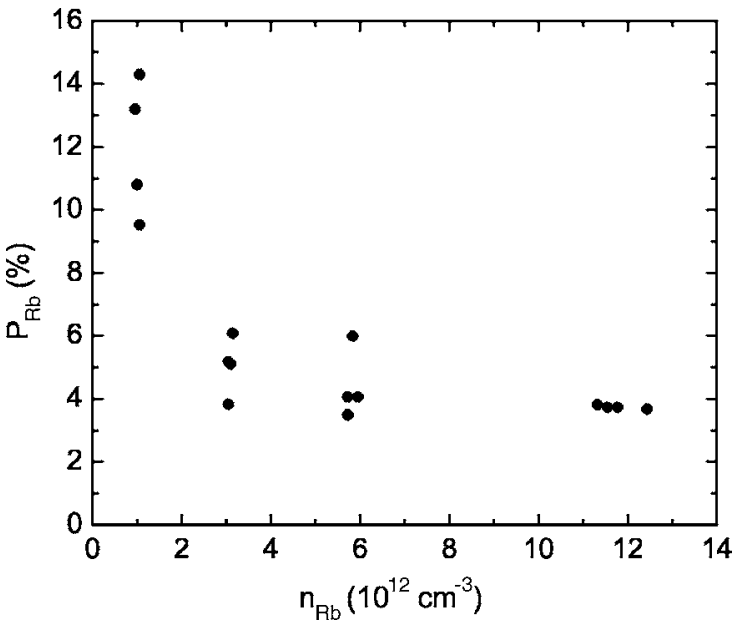

FIG. 3. Rubidium polarization versus rubidium density with no buffer gas present.

level system. If the sample is a sphere of radius $R$, and the populations of the ground and excited states are spatially constant, the rate equation for the ground-state atoms at the center of the sphere is

$$
\begin{aligned}
\frac{\partial n_{\mathrm{g}}}{\partial t}= & \alpha\left(n_{\mathrm{e}}-n_{\mathrm{g}}\right)+\tilde{\gamma} n_{\mathrm{e}}-\tilde{\gamma} n_{\mathrm{e}} \int_{r=0}^{R} d^{3} r \int_{\nu=0}^{\infty} d \nu \frac{g(\nu)}{4 \pi r^{2}} \\
& \times \exp \left[-\frac{\lambda^{2} A}{8 \pi} g(\nu)\left(n_{\mathrm{g}}-n_{\mathrm{e}}\right) r\right]\left\{\frac{\lambda^{2} A}{8 \pi} g(\nu)\left(n_{\mathrm{g}}-n_{\mathrm{e}}\right)\right\} .
\end{aligned}
$$

Here, the optical pump rate of atoms is $\alpha$, the spontaneous rate of decay of atoms from the excited state to the ground state is $\widetilde{\gamma}$ or $A$, and $g(\nu)$ is the normalized line shape of the transition. In this expression, the first term represents the atoms absorbing light and moving from the ground state to the excited state less those making the opposite transition via stimulated emission. The second term represents the spontaneous decay of excited state atoms. The third term describes the effect of radiation trapping on the number density of ground state atoms at the center of the volume. At a distance $r$ from the center of the cell, the number density of photons produced by spontaneous decay is given by $\widetilde{\gamma} n_{\mathrm{e}}$, and their spatial and frequency distribution is $g(\nu) / 4 \pi r^{2}$. The exponential factor describes the absorption of light as it travels to the center of the volume, and the term in curly brackets the probability that, once at the center, the photons are absorbed. When integrated over $r$ and $\nu$, the third term is the number density of ground state atoms at the center of the volume moved to the excited state by photons emitted by other atoms in the sample.

To evaluate Eq. (7), the volume integral over $r$ is evaluated first, yielding

$$
\begin{aligned}
\frac{\partial n_{\mathrm{g}}}{\partial t}= & \alpha\left(n_{\mathrm{e}}-n_{\mathrm{g}}\right)+\tilde{\gamma} n_{\mathrm{e}}-\tilde{\gamma} n_{\mathrm{e}} \int_{\nu=0}^{\infty} d \nu(g(\nu)-g(\nu) \\
& \left.\times \exp \left[-\frac{\lambda^{2} A R}{8 \pi}\left(n_{\mathrm{g}}-n_{\mathrm{e}}\right) g(\nu)\right]\right) .
\end{aligned}
$$

Since the line shape is normalized, the first term in the inte- gral yields a value of 1 . For the second term, the absorption profile is approximated with a Gaussian line shape with center frequency $\nu_{\mathrm{o}}$ and full width at half maximum (FWHM) $\Delta \omega$, so that

$$
g(\nu)=\frac{2}{\Delta \omega} \sqrt{\frac{\ln 2}{\pi}} \exp \left\{-\frac{4 \ln 2\left(\nu-\nu_{\mathrm{o}}\right)^{2}}{\Delta \omega^{2}}\right\} .
$$

Defining $x^{2}$ to be

$$
\frac{4 \ln 2\left(\nu-\nu_{\mathrm{o}}\right)^{2}}{\Delta \omega^{2}}
$$

the rate equation becomes

$$
\begin{aligned}
\frac{\partial n_{\mathrm{g}}}{\partial t}= & \alpha\left(n_{\mathrm{e}}-n_{\mathrm{g}}\right)+\tilde{\gamma} n_{\mathrm{e}} \int_{x=-\nu_{\mathrm{o}} 2 \sqrt{\ln 2 / \Delta \omega}}^{\infty} d x \frac{e^{-x^{2}}}{\sqrt{\pi}} \\
& \times \exp \left[-\frac{\lambda^{2} A R\left(n_{\mathrm{g}}-n_{\mathrm{e}}\right) \sqrt{\ln 2}}{4 \pi \sqrt{\pi} \Delta \omega} e^{-x^{2}}\right], \\
\equiv & \alpha\left(n_{\mathrm{e}}-n_{\mathrm{g}}\right)+\frac{\tilde{\gamma} n_{\mathrm{e}}}{M},
\end{aligned}
$$

where the multiplicity factor, $M$, is defined as the reciprocal of the integral. Comparing Eq. (10) to the rate equation for the same system in the absence of radiation trapping,

$$
\frac{\partial n_{\mathrm{g}}}{\partial t}=\alpha\left(n_{\mathrm{e}}-n_{\mathrm{g}}\right)+\tilde{\gamma} n_{\mathrm{e}}
$$

we see that the expressions differ only by the multiplicity factor in the spontaneous decay term.

The integral is evaluated by extending the lower limit of integration to negative infinity and using Hermite integration, in which an integral of the form $\int_{-\infty}^{+\infty} F(x) e^{-x^{2}} d x$ is approximated with $\sum_{i} w_{i} F\left(\chi_{i}\right)$. The factors $w_{i}$ and $\chi_{i}$ are the weights and abscissas for Hermite integration [19]. Factors for Hermite integration can be found in standard references; for our symmetric integral we used values for 32-term point Hermite integration from an engineering website [20], yielding a 16-term sum for $M$. Approximating the difference of the number densities of atoms in the ground and excited states as the overall number density gives, for our experiment,

$$
M=\frac{\sqrt{\pi}}{\sum_{\mathrm{i}} w_{i} \exp \left\{-\frac{n_{\mathrm{Rb}} R \lambda_{1}{ }^{2} A_{1} \sqrt{\ln 2}}{\Delta \nu_{1} 4 \pi \sqrt{\pi}} \exp \left[-\chi_{i}^{2}\right]\right\}} .
$$

Here, $\lambda_{1}$ is the wavelength of the $D_{1}$ transition, $A_{1}$ is the spontaneous decay rate, and $\Delta \nu_{1}$ is the full width of the transition.

In the regime of $n_{\mathrm{Rb}}=10^{12}-10^{13} \mathrm{~cm}^{-3}$, our variation of $10-20 \%$ in the Rb number density translates to a variation of $10-30 \%$ in the value of $(M-1)$. We use values of $795 \mathrm{~nm}, 3.6 \times 10^{7} \mathrm{~Hz}$, and $1.75 \mathrm{~cm}$ for $\lambda_{1}, A_{1}$, and $R$, respectively. Numerically we find that $M=3.4$ at a rubidium density of $1.0 \times 10^{12} \mathrm{~cm}^{-3}, \quad M=14$ at $3.0 \times 10^{12}, \quad M=28$ at 
$5.1 \times 10^{12} \mathrm{~cm}^{-3}$, and $M=63$ at $1.0 \times 10^{13} \mathrm{~cm}^{-3}$.

The effects of pressure broadening are not accounted for in our model; we simply fix the absorption width of the Rb $D 1$ transition as $8 \mathrm{GHz}$. Since absorption extends well into the wings of each of the individual hyperfine transitions of each isotope, this seems to be an acceptable approximation. (All four of the buffer gases we employed broaden the $\mathrm{Rb}$ transition at about the same rate of $29 \mathrm{MHz} /$ torr for $100{ }^{\circ} \mathrm{C}$ $[21,22])$.

If a photon from an optical pumping cycle is reabsorbed $(M-1)$ number of times and the optical pump rate is $\gamma$, to first order, the contribution of radiation trapping to spin relaxation is

$$
\Gamma_{\mathrm{rt}}=K(M-1) \gamma,
$$

where $K$ is a fit parameter. We expect $K$ to be less than 1.0 because scattered photons may be less than perfectly depolarizing and the nuclear spin may have an inertial effect on the radiation trapping, as described by a "nuclear slowingdown factor," $S$ [23]. Equation (13), however, neglects the quenching effect of the molecular buffer gases.

The atomic buffer gases, argon and helium, and the molecular buffer gases, nitrogen and hydrogen, are both useful in reducing the rate of depolarization due to diffusion to the walls. Collisions with atomic buffer gases will not change the electronic state of the rubidium, but a collision with a molecule can de-excite the rubidium, transferring the energy into the internal vibrational states of the molecule. Since this prevents a photon from being spontaneously emitted, this phenomenon is referred to as quenching. It is standard procedure in spin-exchange optical pumping to include a quantity of $\mathrm{N}_{2}$ (at least 50 torr) in the optical pumping volume for precisely this reason.

The rate of quenching collisions, $\Gamma_{\mathrm{Q}}$, can be determined from the quenching cross section, $\sigma_{\mathrm{Q}}$, the relative velocity of the two species $v_{\text {rel }}$ [24], and the number density of buffergas atoms, $n_{\text {buf: }}$

$$
\Gamma_{\mathrm{Q}}=n_{\text {buf }}\left\langle\sigma_{\mathrm{Q}} v_{\text {rel }}\right\rangle .
$$

Quenching cross sections for the rubidium ${ }^{2} P_{1 / 2} \rightarrow{ }^{2} S_{1 / 2}$ transitions have been measured [25] to be $5.8 \times 10^{-15} \mathrm{~cm}^{2}$ for nitrogen and $6 \times 10^{-16} \mathrm{~cm}^{2}$ for hydrogen, and the relative velocities between rubidium and the buffer-gas molecules is $5.8 \times 10^{4} \mathrm{~cm} / \mathrm{s}$ for nitrogen and $1.9 \times 10^{5} \mathrm{~cm} / \mathrm{s}$ for hydrogen. If $A_{1}$ is the spontaneous decay rate, the fraction of atoms that reach the ground state by emitting a photon rather than quenching is

$$
f_{\text {spon }}=\frac{A_{1}}{\mathrm{~A}_{1}+\Gamma_{\mathrm{Q}}} .
$$

Evaluating Eq. (15) and expressing it in more convenient units gives

$$
f_{\text {spon, } \mathrm{H}_{2}}=\frac{12 \text { torr }}{12 \text { torr }+p_{\mathrm{H}_{2}}}
$$

and

$$
f_{\text {spon, } \mathrm{H}_{2}}=\frac{4.1 \text { torr }}{4.1 \text { torr }+p_{\mathrm{N}_{2}}},
$$

where $p$ stands for the buffer gas pressure in torr at $100{ }^{\circ} \mathrm{C}$.

We can now modify Eq. (13) to express the contribution of radiation trapping, including quenching, to the spin relaxation rate,

$$
\Gamma_{\mathrm{rt}}=K(M-1) \gamma f_{\text {spon }},
$$

where $f_{\text {spon }}$ is given in Eqs. (16a) and (16b) for the molecular buffer gases and $f_{\text {spon }}=1$ for the atomic buffer gases.

To complete our model, we consider the optical pumping rate, $\gamma$. This is a two-stage process of photon absorption at rate $\alpha$ and subsequent spontaneous decay at rate $A_{1}$. In the presence of buffer gas, the upper states of the rubidium mix rapidly, giving equal populations of the excited state. Thus, we write

$$
\frac{1}{\gamma_{\text {untrapped }}}=2\left(\frac{1}{\alpha}+\frac{1}{A_{1}}\right) .
$$

This expression is modified by radiation trapping to be

$$
\frac{1}{\gamma_{\text {trapped }}}=2\left(\frac{1}{\alpha}+\frac{M}{A_{1}}\right) .
$$

In this experiment, the photon absorption rate is several orders of magnitude below the spontaneous decay rate, while $M$ remains less than 100 . We can therefore neglect the second term in the calculation of the optical pumping rate, and simply write $\gamma=\alpha / 2$.

Strictly speaking, both $\gamma$ and the spin destruction rate $\Gamma$ depend on position and frequency in a complicated fashion. For purposes of our simplified model, however, we will take both $\gamma$ and $\Gamma$ to be averages over space and wavelength. Likewise the nuclear slowing-down factor $S$ [23] will affect the optical pumping rate, but since we treat $\gamma$ as a fit parameter this does not affect the model.

Substituting Eqs. (17) and (5) into Eq. (4) gives

$$
P=\frac{1}{1+\frac{\Gamma_{\mathrm{dif}}}{\gamma}+K(M-1) f_{\mathrm{spon}}} .
$$

This function is plotted with the data in Figs. 2(a)-2(h) using our best fit values. We use $K=0.12$ for all plots, but vary $\gamma$ with $\mathrm{Rb}$ density; $\gamma=500 \mathrm{~s}^{-1}$ for the lowest density [plots (a) and (e)], $325 \mathrm{~s}^{-1}$ for the second lowest [(b) and (f)], $200 \mathrm{~s}^{-1}$ for the second highest $[(\mathrm{c})$ and $(\mathrm{g})]$ and $150 \mathrm{~s}^{-1}$ for the highest density $[(\mathrm{d})$ and (h)]. These values fall with density as expected, given the increasing loss of pumping light at the front of the cell.

\section{DISCUSSION AND CONCLUSIONS}

Examining the figures, one sees that our empirical model captures many features of the data. For low buffer-gas pressures, the polarizations increase with buffer-gas pressure because the buffer gases inhibit diffusion to the walls. $\mathrm{Ru}-$ bidium polarizations for nitrogen and argon are very similar 
in this regime, as would be expected from the similarity of their diffusion constants. Rubidium polarization with hydrogen buffer gas is less than that with helium, again as expected from the larger diffusion constant of $\mathrm{H}_{2}$. After a slow initial rise, however, the curves for molecular buffer gases diverge from those of the atomic buffer species. The molecular curves diverge from the atomic ones at 1 or 2 torr for nitrogen versus argon, and closer to 5 torr for hydrogen and helium. In each case, this is the value for which $f_{\text {spon }}$ reaches about $2 / 3$.

As the molecular buffer-gas pressure increases further, the $\mathrm{Rb}$ polarization rises steadily; for nitrogen at the higher rubidium densities the values actually rise faster than our model prediction. In contrast, for increasing amounts of atomic buffer gas the polarization curves flatten out when the radiation trapping term of Eq. (20) exceeds the diffusion term. As the $\mathrm{Rb}$ number density increases this occurs at a lower buffer-gas pressure, and so results in a lower polarization plateau. For the lower rubidium densities, the polarization of the atomic species actually falls in the higher pressure region. Since this only happens to the atomic species, we speculate that this is related to the intricacies of the radiation trapping effects. The details of that process are not obvious to us, and are not reflected in our model. As we mentioned previously, depolarization at higher atomic buffer-gas densities due to depolarizing collisions with the buffer gas itself can safely be ruled out based on known rates for both binary [9] and three-body collisions [10].

Despite the multitude of approximations made, Eq. (20) serves fairly well as a simple model of rubidium polarization for low buffer-gas pressures, as can be seen by the rough agreement with the data. The virtue of the model is that it provides an estimate of rubidium polarization under these complicated conditions, with only a few known or calculated values required as input.

In conclusion, we have presented data sets for rubidium polarization as a function of alkali density and buffer gas pressure. Radiation trapping is the limiting factor for many of our polarizations, and quenching effects are clearly visible in the contrast between atomic and molecular species of similar sizes. We have developed a simplified model that gives rough agreement with the data and provides further insight into the process of radiation trapping.

\section{ACKNOWLEDGMENTS}

The authors gratefully acknowledge useful discussions with Herman Batelaan and Will Happer. Maya Fabrikant and Josh Machacek provided tremendous help with figure presentation. This work was supported by NSF Grant No. PHY0354946. Los Alamos Unlimited Release number LA-UR06-6659.
[1] D. F. Phillips, A. Fleischhauer, A. Mair, R. L. Walsworth, and M. Lukin, Phys. Rev. Lett. 86, 783 (2001).

[2] M. Hashimoto and M. Ohtsu, IEEE J. Quantum Electron. 23, 446 (1987).

[3] Y.-Y. Jau, A. B. Post, N. N. Kuzma, A. M. Braun, M. V. Romalis, and W. Happer, Phys. Rev. Lett. 92, 110801 (2004).

[4] T. G. Walker and W. Happer, Rev. Mod. Phys. 69, 629 (1997).

[5] H. Batelaan, A. S. Green, B. A. Hitt, and T. J. Gay, Phys. Rev. Lett. 82, 4216 (1999).

[6] N. Andersen and K. Bartschat, Polarization, Alignment and Orientation in Atomic Collisions (Springer-Verlag, New York, 2001).

[7] R. J. Knize, Z. Wu, and W. Happer, Adv. At. Mol. Phys. 24, 223 (1987)

[8] Z. Wu, M. Kitano, W. Happer, M. Hou, and J. Daniels, Appl. Opt. 25, 4483 (1986).

[9] F. A. Franz and C. Volk, Phys. Rev. A 14, 1711 (1976).

[10] M. A. Bouchiat, J. Brossel, and L. C. Pottier, J. Chem. Phys. 56, 3703 (1972).

[11] M. E. Wagshul and T. E. Chupp, Phys. Rev. A 49, 3854 (1994).

[12] M. Aymar, M. A. Bouchiat, and J. Brossel, J. Phys. (Paris) 30, 619 (1969)
[13] R. J. McNeal, J. Chem. Phys. 37, 2726 (1962).

[14] D. Tupa, L. W. Anderson, D. L. Huber, and J. E. Lawler, Phys. Rev. A 33, 1045 (1986).

[15] D. Tupa and L. W. Anderson, Phys. Rev. A 36, 2142 (1987).

[16] A. F. Molisch, B. P. Oehry, W. Schupita, and G. Magerl, J. Phys. B 30, 1879 (1997).

[17] J. E. Lawler, J. J. Curry, and G. G. Lister, J. Phys. D 33, 252 (2000).

[18] D. Tupa (unpublished).

[19] M. Abramowitz and I. A. Stegun, Handbook of Mathematical Functions with Formulas, Graphs, and Mathematical Tables (Dover, New York, 1970).

[20] http://www.efunda.com/math/num_integration/ findgausshermite.cfm

[21] S.-Y. Ch'en and M. Takeo, Rev. Mod. Phys. 29, 20 (1957).

[22] M. V. Romalis, E. Miron, and G. D. Cates, Phys. Rev. A 56, 4569 (1997).

[23] M. E. Wagshul and T. E. Chupp, Phys. Rev. A 40, 4447 (1989).

[24] F. Masnou-Seeuws and M.-A. Bouchiat, J. Phys. (Paris) 28, 406 (1967).

[25] E. S. Hrycyshyn and L. Krause, Can. J. Phys. 48, 2761 (1970). 